\title{
Existence of the core in a heterogeneous divisible commodity exchange economy
}

\author{
Farhad Hüsseinov
}

Accepted: 20 February 2008 / Published online: 11 March 2008

(C) Springer-Verlag 2008

\begin{abstract}
We consider the exchange of a heterogeneous divisible commodity modeled as a measurable space. Under rational, continuous and convex preferences over characteristic measures a weak core is shown to exist. Further, a core exists if characteristic measures are mutually absolutely continuous. Applied to the land trading economy, the core existence results in Berliant (J Math Econ 14:53-56, 1985) and Dunz (Reg Sci Urban Econ 21:73-88, 1991) are obtained.
\end{abstract}

Keywords Measurable space exchange economy $\cdot$ Core $\cdot$ NTU game

\section{Introduction}

We consider the problem of exchange of a heterogeneous divisible commodity. One notable example of such a commodity is land. This problem is coined in the literature as the "cake division" or "land trading" problem. A heterogeneous divisible commodity is modeled as a measurable space $(X, \Sigma)$. In theoretical models of land economics, $X$ is assumed to be a Borel measurable subset of Euclidean space $R^{2}$ (or more generally $R^{k}$ ), and $\Sigma$ to be the Borel $\sigma$-algebra $\mathcal{B}(X)$ of subsets of $X$. It is usual to consider this measurable space with the Lebesgue measure.

\footnotetext{
The author is grateful to the participants of economic theory seminars at Bilkent University and participants of the session in the 17th International Conference on Game Theory at Stony Brook, July 10-14, 2006, where results of this paper were presented, for useful discussions and the referees for comments that led to a number of improvements. I thank Drs. Nedim Alemdar and Refet Gürkaynak for useful discussions. Special thanks go to Professor William Thomson and Özgür Evren.
}

\section{F. Hüsseinov $(\varangle)$}

Department of Economics, Bilkent University, 06800 Bilkent, Ankara, Turkey

e-mail: farhad@bilkent.edu.tr 
Berliant (1985) is the first to study the core in the context of a land trading economy. He shows the existence of a competitive equilibrium when preferences over plots are represented by measures that are absolutely continuous with respect to the Lebesgue measure. He derives the core existence result from the existence of a competitive equilibrium in a land trading economy, which is the main result of his paper (Berliant 1985).

Dunz (1991) studies the existence of the core also in the context of a land trading economy, but for substantially more general preferences. In Dunz (1991) preferences are represented by utility functions that are compositions of quasi-concave functions with a finite number of characteristics of parcels. These characteristics are countably-additive measures over parcels. Dunz proves that under these assumptions on preferences and the assumption of absolute continuity of chracteristic measures with respect to the Lebesgue measure, the weak core of a land trading economy is nonempty. Assigning a finite number of additive characteristics to parcels is a common assumption made in empirical literature on land trading. Dunz's proof of the existence of the weak core is direct, and uses Scarf's theorem on the existence of the weak core in nontransferable utility games and a limiting procedure.

Berliant and Dunz (2004) (BD hereafter) lay the foundations of the general equilibrium theory for a very general land trading economy adopting preferences over parcels which take into account their geometric form. BD's core existence result (Theorem 4) assumes local nonsatiation, local nonminimization and existence of nonwasteful partitions for preferences (For the definitions of the last two properties see BD pages 600 and 605). The main result of the present paper-the weak core existence-does not make any of these assumptions. However, in proving the coincidence of the weak core and the core, we assume mutual absolute continuity of the individual characteristic vector-measures and monotonicity of preferences, which together imply nonwasteful partitions property. BD [Sect. 5.1] considers a model of exchange similar to that of the present paper in the context of land exchange. They assume quasi-concave utility functions of class $C^{1}$ over characteristic measures. Their core existence result (Theorem 4) applied to this model requires the partial derivatives of utility functions to be positive and uniformly bounded away from zero and infinity. In economic terms, this amounts to assuming that the marginal rates of substitution between the characteristics of parcels are uniformly bounded away from zero and infinity (In this regard see also the definition of separation by hyperplanes assumption in BD, pages 600601). In contrast, we admit arbitrary continuous quasi-concave utility functions over characteristic measures.

A related work is due to Weller (1985) who consider the problem of fair division of a measurable space $(X, \Sigma)$ under the assumption that each agent's preferences over measurable subsets are represented by a countably additive measure, and shows the existence of an envy-free and efficient partition in this problem.

In this paper we prove the existence of the core in a general context of a measurable space trading economy. Applied to the land trading economy it gives Dunz's core existence result. 


\section{The model and preliminaries}

Let $(X, \Sigma)$ be a measurable space (the "cake" or "land plot") and let $\bar{P}=$ $\left\{A_{1}, A_{2}, \ldots, A_{n}\right\}$ be a measurable ordered partition of $X$. Let $\mu_{1}, \mu_{2}, \ldots, \mu_{n}$ be nonatomic finite vector measures on $(X, \Sigma)$ of dimension $s_{1}, s_{2}, \ldots, s_{n}$, respectively. All measures in this paper are assumed to be countably additive.

The interpretation is that there are $n$ persons $(i=1,2, \ldots, n)$ each contributing his share $A_{i}(i \in N)$ of the whole cake $X$, and pieces of this cake are valued by individuals according to their measures $\mu_{1}, \mu_{2}, \ldots, \mu_{n}$, respectively. The components of vectormeasure $\mu_{i}(B)$ are interpreted as measures of different attributes of a measurable piece $B$ attached to this piece by individual $i$. We assume that individual $i$ has a preference $\succ_{i}$ over his subjective attributes' profiles $\mu_{i}(B), B \in \Sigma$ and hence over measurable sets $B \in \Sigma$. We will use the same symbol $\succ_{i}$ for denoting both of these preferences. No confusion should arise. Every ordered measurable partition $\left\{B_{1}, B_{2}, \ldots, B_{n}\right\}$ will be interpreted as a feasible allocation of $X$. In the sequel, all partitions that we consider are assumed to be ordered and measurable.

A coalition is an arbitrary nonempty subset of $N=\{1, \ldots, n\}$. The set of all coalitions is denoted as $\mathcal{N}$.

Definition 1 A coalition $I \subset N$ improves (weakly improves) upon a partition $P=$ $\left\{B_{1}, B_{2}, \ldots, B_{n}\right\}$ if there exists a partition $Q=\left\{C_{i} \mid i \in I\right\}$ of $A(I)=\cup_{i \in I} A_{i}$ such that $C_{i} \succ_{i} B_{i}$ for all $i \in I$ (not $B_{i} \succ_{i} C_{i}$ for all $i \in I$ and $C_{i} \succ_{i} B_{i}$ for at least one $i \in I$.)

Definition 2 Partition $P=\left\{B_{1}, B_{2}, \ldots, B_{n}\right\}$ is a weak core partition (core partition) if no coalition improves (weakly improves) upon partition $P$. The set of all weak core partitions is the weak core (core) of the measurable space partition problem.

We will formulate two facts that play central roles in the proofs. We will identify a vector of vectors (perhaps of different dimensions) as a long vector with scalar coordinates arranged in the lexicographic order. Sometimes we will denote coordinates with double indexes, with the first index being the index of a component vector and the second one the index of a component in that component vector.

The following proposition is a generalization of a result known as Dubins-Spanier's theorem (see also Aliprantis and Border 1994, p. 358) and easily follows from that result. It is to be noted that, in fact, this theorem was discovered a decade earlier than Dubins and Spanier by Dvoretsky et al. (1951) (see also Chernoff 1951) and is a consequence of the celebrated Liapunov convexity theorem (Liapunov 1940).

Proposition 1 Let $(X, \Sigma)$ be a measurable space and let $\mu_{1}, \mu_{2}, \ldots, \mu_{n}$ be nonatomic finite vector measures on $(X, \Sigma)$ of dimensions $s_{1}, s_{2}, \ldots, s_{n}$, respectively. Then, the following set in $R^{s}$, where $s=\sum_{j=1}^{n} s_{j}$,

$$
\mathcal{R}=\left\{\left(\mu_{i}\left(B_{i}\right)\right)_{i=1}^{n} \in R^{s} \mid P=\left\{B_{1}, B_{2}, \ldots, B_{n}\right\} \text { is a partition of } X\right\}
$$


Proof of Proposition 1 is based on Dubins-Spanier's theorem. Let $\mu=\left(\mu^{k}\right)_{k=1}^{s}$ be a vector measure $\left(\mu_{1}, \mu_{2}, \ldots, \mu_{n}\right)$ of dimension $s$. With every partition $P=$ $\left\{B_{1}, B_{2}, \ldots, B_{n}\right\}$ we associate the $s \times n$ matrix of reals $M(P)=\left(\mu^{k}\left(B_{i}\right)\right)$. Denote by $M^{s \times n}$ the space of all $s \times n$ matrices with real entries. By Proposition 1 in Dubins and Spanier (1961) the range $\mathcal{R}^{\prime} \subset M^{s \times n}$ of matrix-valued function $M$ is compact and convex.

Let $L: M^{s \times n} \rightarrow R^{s}$ be a mapping defined in the following way. The first $s_{1}$ components of $L(M)$ are the first $s_{1}$ entries in the first column of matrix $M$, the second $s_{2}$ components are the entries in the second column of $M$ with the row indexes $s_{1}+1$ through $s_{1}+s_{2}$, and so on. Clearly $L$ is a linear mapping with $L\left(\mathcal{R}^{\prime}\right)=\mathcal{R}$. Since $\mathcal{R}^{\prime}$ is compact and convex it follows that so is $\mathcal{R}$.

Corollary 2 ("Generalization” of Dubins-Spanier's Theorem). Let $(X, \Sigma)$ be a measurable space and let $\mu_{1}, \mu_{2}, \ldots, \mu_{n}$ be nonatomic finite vector measures on $(X, \Sigma)$ of the same dimension $l$. Then the following set

$M(P)=\left\{\left(\mu_{1}\left(B_{1}\right), \mu_{2}\left(B_{2}\right), \ldots, \mu_{n}\left(B_{n}\right)\right) \mid P=\left\{B_{1}, B_{2}, \ldots, B_{n}\right\}\right.$ is a partition of $\left.X\right\}$

in $M^{l \times n}$ is compact and convex.

In fact, Proposition 1 and Corollary 2 can be considered as versions of DubinsSpanier's Theorem (Dubins and Spanier 1961), that turn out to be convenient in different contexts.

Another important fact is Scarf's theorem on the existence of the weak core in nontransferable utility (NTU) games. We first recall the concept of an NTU game. Let, as before, $N=\{1, \ldots, n\}$ be the set of all players. For each coalition $S$ a set $V(S)$ in $R^{n}$ is given. It is assumed that this set is nonempty closed comprehensive and satisfies the following condition: if $x \in V(S)$ and $y \in R^{n}$ are such that $x_{i}=y_{i}$ for all $i \in S$, then $y \in V(S)$. Comprehensiveness means that if $x \in V(S)$ and $x \geqq y$ then $y \in V(S)$. Vectors from $V(S)$ are interpreted as utility profiles available to members of the coalition $S$. An NTU game is a pair $(N, V)$ where $V$ is a mapping from $\mathcal{N}$ into subsets of $R^{n}$. Denote by int $V(S)$ the interior of $V(S)$. A utility profile $x \in V(N)$ is a weak core profile if $x \notin$ int $V(S)$ for all coalitions $S$. That is, if there is no utility profile $x^{\prime} \in V(S)$ that gives each member of coalition $S$ more utility than $x$. The weak core is the set of all weak core profiles.

A collection of coalitions $\mathcal{B}$ is balanced if there are weights $\delta_{S} \geq 0(S \in \mathcal{B})$ such that $\sum_{S \in \mathcal{B}_{i}} \delta_{S}=1$ for every $i \in N$. Here $\mathcal{B}_{i}=\{S \in \mathcal{B} \mid S \ni i\}$. A game $(N, V)$ is balanced if the inclusion

$$
\cap_{S \in \mathcal{B}} V(S) \subset V(N)
$$

holds for an arbitrary balanced collection of coalitions $\mathcal{B}$.

Theorem 3 (Scarf 1967). A balanced NTU game has a nonempty weak core.

Scarf (1967) also shows that nonemptiness of the weak core of a classical exchange economy with a finite number of homogeneous divisible commodities and convex preferences is a direct consequence of this theorem. 
However, Theorem 3 does not immediately apply to the model of exchange considered in this paper. The model of exchange considered here contains an uncountable infinity of commodities, each of which is available exactly in one unit. The commodity space is the $\sigma$-algebra $\Sigma$ of measurable sets of a measurable space $(X, \Sigma)$. In contrast with the classical model, where the commodity space is the nonnegative orthant of a Euclidean space, the commodity space here has no linear or convex structure. Accordingly, the proof of the nonemptiness of the weak core for this model requires more elaborate techniques. For example, closedness of sets $V(S)$ in the case of classical exchange model is a direct consequence of the continuity of preferences. Whereas in the present model, the proof of this property makes use of the compactness statement in Dubins-Spanier's Theorem.

\section{The main result}

We will assume that preferences $\succ_{i}(i \in N)$ over characteristic measures are rational, continuous and convex, so that they can be represented by continuous quasiconcave utility functions $u_{i}(i \in N)$ defined on the space of characteristics $R_{+}^{s_{i}}(i \in N)$. We will represent preferences over $\Sigma$ by introducing utility functions $U_{i}(B)=u_{i}\left(\mu_{i}(B)\right)(i \in$ $N)$. Thus, in addition to the assumptions of the previous section here we assume rationality (that is, asymmetry and negative transitivity) of preferences.

For a (weak) core partition $P=\left\{B_{1}, B_{2}, \ldots, B_{n}\right\}$ the utility profile $\left(U_{1}\left(B_{1}\right)\right.$, $\left.U_{2}\left(B_{2}\right), \ldots, U_{n}\left(B_{n}\right)\right)$ will be called a (weak) core utility profile.

Theorem 4 If measures $\mu_{i}(i \in N)$ are nonatomic and utility functions $u_{i}(i \in N)$ are continuous and quasi-concave, then the weak core of the measurable space trading economy is nonempty.

Proof The proof will use Proposition 1 and Scarf's theorem formulated in the previous section.

For each coalition $S$ define a subset $V(S)$ of $R^{n}$ in the following way. Consider all partitions $P=\left\{C_{1}, C_{2}, \ldots, C_{n}\right\}$ of $\cup_{i \in S} A_{i}$, where $C_{j}=\emptyset$ for all $j \notin S$. Set $x(P)=\left(U_{1}\left(C_{1}\right), U_{2}\left(C_{2}\right), \ldots, U_{n}\left(C_{n}\right)\right) \in R^{n}, \quad A(S)=\cup_{i \in S} A_{i}$ and

$$
V_{0}(S)=\{x(P) \mid P \text { is a partition of } A(S)\} .
$$

We define $V(S)$ as the smallest comprehensive set in $R^{n}$ containing $V_{0}(S)$ and with the property: if $x \in V(S)$ then $x^{\prime} \in V(S)$, if $x_{j}^{\prime}=x_{j}$ for all $j \in S$. That is

$$
V(S)=V_{0}(S)+R^{S^{c}}+R_{-}^{n},
$$

where $S^{c}=N \backslash S$ and $R^{S^{c}}$ is the coordinate subspace in $R^{n}$ whose coordinates have as subscripts the players in $S^{c}$. By Proposition 1 the set $\left\{\left(\mu_{i}\left(C_{i}\right)\right)_{i \in S} \mid\left\{C_{1}, C_{2}, \ldots, C_{n}\right\}\right.$ is a partition of $A(S)$ with $C_{j}=\varnothing$ for all $\left.j \notin S\right\}$ is compact. Continuity of functions $u_{i}(i \in N)$ imply that set $V_{0}(S)$ is compact. Compactness of $V_{0}(S)$ in turn, implies the closedness of $V(S)$. So, sets $V(S)$ are closed for all $S \in \mathcal{N}$. 
Thus, we have defined an NTU game $(N, V)$. The weak core, $W C(N, V)$, of this game is defined as the set of all vectors from $V(N) \backslash \cup_{S \in \mathcal{N}}$ int $V(S)$. We will show that nonemptiness of the weak core of this game implies the nonemptiness of the weak core of the measurable space exchange economy. Let $x \in W C(N, V)$. Then by the definition of set $V(N)$, there exists $\bar{x} \in V_{0}(N)$ with $\bar{x} \geqq x$. Since $\bar{x} \in V_{0}(N)$, there exists a partition $P=\left\{B_{1}, B_{2}, \ldots, B_{n}\right\}$ of $X$ such that $\bar{x}=\left(U_{i}\left(B_{i}\right)\right)_{i=1}^{n}$. We assert that $P$ belongs to the weak core of the measurable space exchange economy. Indeed, if a coalition $S$ improves upon partition $P$, then there exists a partition $\left\{C_{i} / i \in S\right\}$ of $A(S)$ such that $U_{i}\left(C_{i}\right)>U_{i}\left(B_{i}\right)=\bar{x}_{i}$ for $i \in S$. By the definition of $V(S)$, then int $V(S) \ni \bar{x}$, and hence, since $V(S)$ is comprehensive int $V(S) \ni x$. This contradicts $x \in W C(N, V)$. Thus, if we show that the weak core of the NTU game $(N, V)$ is nonempty we are done.

According to Scarf's theorem if for an arbitrary balanced collection of coalitions $\mathcal{B}$ the inclusion stop

$$
\cap_{S \in \mathcal{B}} V(S) \subset V(N)
$$

holds, then the weak core of an NTU game is nonempty. Thus, if we show that inclusion (1) holds, then the theorem will be proved.

Let $\mathcal{B}$ be a balanced collection of coalitions with the balancing weights $\delta_{S} \geq$ $0, S \in \mathcal{B}$. Let $x \in \cap_{S \in \mathcal{B}} V(S)$. For every coalition $S \in \mathcal{B}$ the set $A(S)=\cup_{i \in S} A_{i}$ has a partition $P_{S}=\left\{A^{1}(S), A^{2}(S), \ldots, A^{n}(S)\right\}$ such that

$$
U_{j}\left(A^{j}(S)\right) \geq x_{j} \text { for all } j \in S \text { and } A^{j}(S)=\emptyset \text { for all } j \notin S \text {. }
$$

Denote

$$
A_{i}^{j}(S)=A_{i} \cap A^{j}(S) \text { for } i, j \in N
$$

Clearly, for each pair $S \in \mathcal{B}$ and $i \in N$ such that $S \ni i,\left\{A_{i}^{1}(S), A_{i}^{2}(S), \ldots, A_{i}^{n}(S)\right\}$ is a partition of $A_{i}$. For $i, j \in S$ the set $A_{i}^{j}(S)$ can be interpreted as the part of endowment of individual $i, A_{i}$, which $i$ should give to $j$ so that coalition $S$ achieves at least the utility profile $x_{S}=\left(x_{i}\right)_{i \in S}$. Pay attention that if $\{i, j\} \nsubseteq S$, that is, if $i$ or $j$ is not a member of $S$, then $A_{i}^{j}(S)=\emptyset$.

So, for each $i \in N$ we have a collection of partitions $\left\{A_{i}^{1}(S), A_{i}^{2}(S), \ldots, A_{i}^{n}(S)\right\} \mid$ $\left.S \in \mathcal{B}_{i}\right\}$ of set $A_{i}$. Since $\sum_{S \in \mathcal{B}_{i}} \delta_{S}=1$, by Proposition 1 there exists a partition $\left\{A_{i}^{1}, A_{i}^{2}, \ldots, A_{i}^{n}\right\}$ of $A_{i}$ so that

$$
\mu_{j}\left(A_{i}^{j}\right)=\sum_{S \ni j} \delta_{S} \mu_{j}\left(A_{i}^{j}(S)\right) \text { for all } j \in N
$$


Let $B_{j}=\cup_{i \in N} A_{i}^{j}, j \in N$. Then $P=\left\{B_{1}, B_{2}, \ldots, B_{n}\right\}$ is a partition of $X$ and

$$
\begin{aligned}
\mu_{j}\left(B_{j}\right) & =\sum_{i \in N} \mu_{j}\left(A_{i}^{j}\right)=\sum_{i \in N} \sum_{S \ni j} \delta_{S} \mu_{j}\left(A_{i}^{j}(S)\right)=\sum_{S \ni j} \delta_{S}\left[\sum_{i \in N} \mu_{j}\left(A_{i}^{j}(S)\right)\right] \\
& =\sum_{S \ni j} \delta_{S} \mu_{j}\left(A^{j}(S)\right) .
\end{aligned}
$$

The second equality above follows from (4) and the fourth equality from the fact that $\left\{A_{1}^{j}(S), A_{2}^{j}(S), \ldots, A_{n}^{j}(S)\right\}$ is a partition of $A^{j}(S)$.

Since utility functions $u_{i}$ are quasi-concave and $\sum_{S \ni j} \delta_{S}=1$, using inequalities (2) we obtain from (5)

$$
u_{j}\left(\mu_{j}\left(B_{j}\right)\right)=u_{j}\left(\sum_{S \ni j} \delta_{S} \mu_{j}\left(A^{j}(S)\right)\right) \geq \min _{S \ni j} u_{j}\left(A^{j}(S)\right) \geq x_{j} .
$$

So $U_{j}\left(B_{j}\right) \geq x_{j}$ for all $j \in N$. Hence $x \in V(N)$.

\section{Examples and existence of the core}

As was noted in the Introduction, Theorem 3 implies the main result of Dunz (1991). Although, this result claims the existence of a core (rather than a weak core) we give an example demonstrating that under the assumptions made in Dunz (1991) the core may be empty. Moreover, in Dunz (1991) continuity of utility functions is not explicitly assumed. We also give an example to illustrate that without the continuity of utility functions even the weak core may be empty.

Example 1 Let $X=[0,4] \times[0,1], A_{1}=[0,2] \times[0,1], A_{2}=(2,3] \times[0,1], A_{3}=$ $(3,4] \times[0,1]$. Let $\lambda$ denote the 2-dimensional Lebesgue measure. Let utilities be given by measures $\mu_{1}, \mu_{2}, \mu_{3}$ defined as $\mu_{1}=\lambda$ on $[0,1] \times[0,1]$, and 0 on $(1,4] \times$ $[0,1]$, and $\mu_{2}=\mu_{3}=\lambda$ on $[1,4] \times[0,1]$ and $\mu_{2}=\mu_{3}=0$ on $[0,1] \times[0,1]$. Then

$$
\begin{aligned}
& V(1)=(1,-,-), \text { i.e. } V(1)=\left\{x \in R^{3} \mid x_{1} \leq 1\right\}, \\
& V(2)=(-, 1,-), \\
& V(3)=(-,-, 1), \\
& V(12)=(1,2,-), \text { i.e. } V(12)=\left\{x \in R^{3} \mid x_{1} \leq 1, x_{2} \leq 2\right\}, \\
& V(13)=(1,-, 2),
\end{aligned}
$$

$V(23)=\left\{\left(x_{1}, x_{2}, x_{3}\right) \in R^{3} \mid x_{2} \geq 0, x_{3} \geq 0, x_{2}+x_{3}=2\right\}+R_{-}^{3}$, and $V(123)=$ $F+R_{-}^{3}$, where $F=\left\{x \in R^{3} \mid x_{1}=1, x_{2} \geq 0, x_{3} \geq 0, x_{2}+x_{3}=3\right\}$.

The core of this game is empty. The weak core is the set $C=\left\{x \in R^{3} \mid x_{1}=\right.$ $\left.1, x_{2} \geq 1, x_{3} \geq 1, x_{2}+x_{3}=3\right\}$. Indeed, for $x \in C$ a coalition that contains agent 1 has no way of improving the utility of agent 1 , and since for $x \in V(23), x_{2}+x_{3}=2$, the coalition $\{2,3\}$ cannot improve utility profiles in $C$. 
Example 2 Consider $X=[0,2] \times[0,2] \subset R^{2}$ with the Lebesgue measure $\lambda$. Let there be two agents with endowments $A_{1}=[0,1] \times[0,2]$ and $A_{2}=(1,2] \times$ $[0,2]$. Let both agents have the common characteristics $\mu_{1}, \mu_{2}$ defined as $\mu_{i}(B)=$ $\int_{B} h_{i}(x) d \lambda(x)(i=1,2)$ for measurable set $B \subset X$, where $h_{i}(i \in N)$ is the characteristic function of $A_{i}(i \in N)$. Let $u_{1}: R_{+}^{2} \rightarrow R$ be defined as

$$
u_{1}\left(x_{1}, x_{2}\right)= \begin{cases}x_{1} & \text { for } 0 \leq x_{1}, x_{2}<1 \\ 2-x_{1} & \text { for } 1<x_{1} \leq 2,0 \leq x_{2}<1 \\ -1 & \text { elsewhere }\end{cases}
$$

and $u_{2}\left(x_{1}, x_{2}\right)=u_{1}\left(x_{2}, x_{1}\right)$.

It is easily verified that functions $u_{1}, u_{2}$ are quasiconcave. It is easy to calculate $V(1)=\left\{\left(U_{1}, U_{2}\right) \mid U_{1} \leq 0\right\}, \quad V(2)=\left\{\left(U_{1}, U_{2}\right) \mid U_{2} \leq 0\right\}$ and $V(12) \cap R_{+}^{2}=$ $\left\{\left(U_{1}, U_{2}\right): U_{1}<1, U_{2}<1\right\}$. Thus no point in $V(12) \cap R_{+}^{2}$ is a weak core utility profile. Obviously, any point in $R^{2} \backslash R_{+}^{2}$ is blocked by one of the agents. Therefore the weak core and hence the core is empty.

For coincidence of the weak core and the core some further assumptions are required. For vectors $x, y \in R^{k}$ denote $x \geq y$ if $x_{i} \geq y_{i}$ for all $i=1, \ldots, k$ and $x \neq y$. Preference relation $\succ$ defined on $R_{+}^{k}$ will be called monotone if for $x, x^{\prime} \in R_{+}^{k}, x^{\prime} \geq x$ implies $x^{\prime} \succ x$.

Theorem 5 If preference $\succ_{i}$ is rational, continuous and monotone for each $i \in N$, and if the measures $v_{i}=\sum_{j=1}^{s_{i}} \mu_{i}^{j}(i \in N)$ are absolutely continuous with respect to each other, then the weak core and the core coincide. If, in addition, preferences are convex, then the core of the measurable space trading economy is nonempty.

Proof Let $\succcurlyeq_{i}$ be the weak preference relation associated with $\succ_{i}(i \in N)$, and let a coalition $I$ weakly improve upon a partition $P=\left\{B_{1}, B_{2}, \ldots, B_{n}\right\}$ via partition $Q=\left\{C_{i} \mid i \in I\right\}$ of $A(I)=\cup_{i \in I} A_{i}$. So we have, not $B_{i} \succ_{i} C_{i}$ for all $i \in$ $I$ and $C_{i} \succ_{i} B_{i}$ for at least one $i \in I$. Since preferences are assumed to be the strict parts of rational preferences, [not $B_{i} \succ_{i} C_{i}$ ] is equivalent to [ $C_{i} \succcurlyeq_{i} B_{i}$ ]. Let $C_{i_{0}} \succ_{i_{0}} B_{i_{0}}$ for $i_{0} \in I$. Then by the monotonicity assumption, $\mu_{i_{0}}\left(C_{i_{0}}\right) \geq 0$. By the mutual absolute continuity assumption, $\mu_{i}\left(C_{i_{0}}\right) \geq 0$ for all $i \in N$.

By continuity of $\succ_{i_{0}}$ there exists $d>0$ such that for every subset $D \subset C_{i_{0}}$ with $\mu_{i_{0}}(D)<d$, we have $C_{i_{0}} \backslash D \succ_{i_{0}} B_{i_{0}}$. By nonatomicity of measure $\mu_{i_{0}}$, such $D$ exists. Denote $I_{0}=I \backslash\left\{i_{0}\right\}$. By Proposition 1, there exists a partition $D_{i}\left(i \in I_{0}\right)$ of $D$ such that $\mu_{i}\left(D_{i}\right)=\frac{1}{\left|I_{0}\right|} \mu_{i}(D)$ for all $i \in I_{0}$. Define $F_{i_{0}}=C_{i_{0}} \backslash D$, and $F_{i}=$ $C_{i} \cup D_{i}$ for $i \in I \backslash\left\{i_{0}\right\}$. Then $\left\{F_{i} \mid i \in I\right\}$ is a partition of $A(I)$, and by monotonicity of preferences, $F_{i} \succ_{i} C_{i}$ for all $i \in I$. So $I$ improves upon partition $P$.

Thus we have showed that the weak core and the core coincide. This together with Theorem 4 implies the other statement of the theorem. 


\section{References}

Aliprantis C, Border K (1994) Infinite dimensional analysis. Springer, Berlin

Berliant M (1985) An equilibrium existence result for an economy with land. J Math Econ 14:53-56

Berliant M, Dunz K (2004) A foundation of location theory: existence of equilibrium, the welfare theorems, and the core. J Math Econ 40:593-618

Chernoff H (1951) An extension of a result of Liapunoff on the range of a vector measure. Proc Am Math Soc 2:722-726

Dubins LE, Spanier EH (1961) How to cut a cake fairly. Am Math Mon 68:1-17

Dunz K (1991) On the core of a land trading game. Reg Sci Urban Econ 21:73-88

Dvoretsky A, Wald A, Wolfowitz J (1951) Relations among certain ranges of vector measures. Pacific J Math 1:39-74

Liapunov AA (1940) Sur les fonctions-vecteurs completement additives. Bull Acad Sci USSR 4:465-478

Scarf H (1967) The core of an N-person game. Econometrica 35:50-69

Weller D (1985) Fair division of a measurable space. J Math Econ 14:5-17 